\title{
Oxidants, Antioxidants, and the Beneficial Roles of Exercise-Induced Production of Reactive Species
}

\author{
Elisa Couto Gomes, ${ }^{1}$ Albená Nunes Silva, ${ }^{2}$ and Marta Rubino de Oliveira ${ }^{3}$ \\ ${ }^{1}$ Department of Biochemistry and Immunology, Institute of Biological Science, Federal University of Minas Gerais (UFMG), \\ 31270-901 Belo Horizonte, MG, Brazil \\ ${ }^{2}$ Department of Morphology, Institute of Biological Sciences (UFMG); and Santa Casa de Misericórdia, \\ 30150-221 Belo Horizonte, MG, Brazil \\ ${ }^{3}$ School of Sport, Exercise and Health Sciences, Loughborough University, Leicestershire LE11 3TU, UK
}

Correspondence should be addressed to Elisa Couto Gomes, elisacoutogomes@gmail.com

Received 16 March 2012; Accepted 2 April 2012

Academic Editor: Michalis G. Nikolaidis

Copyright ( 2012 Elisa Couto Gomes et al. This is an open access article distributed under the Creative Commons Attribution License, which permits unrestricted use, distribution, and reproduction in any medium, provided the original work is properly cited.

\begin{abstract}
This review offers an overview of the influence of reactive species produced during exercise and their effect on exercise adaptation. Reactive species and free radicals are unstable molecules that oxidize other molecules in order to become stable. Although they play important roles in our body, they can also lead to oxidative stress impairing diverse cellular functions. During exercise, reactive species can be produced mainly, but not exclusively, by the following mechanisms: electron leak at the mitochondrial electron transport chain, ischemia/reperfusion and activation of endothelial xanthine oxidase, inflammatory response, and autooxidation of catecholamines. Chronic exercise also leads to the upregulation of the body's antioxidant defence mechanism, which helps minimize the oxidative stress that may occur after an acute bout of exercise. Recent studies show a beneficial role of the reactive species, produced during a bout of exercise, that lead to important training adaptations: angiogenesis, mitochondria biogenesis, and muscle hypertrophy. The adaptations occur depending on the mechanic, and consequently biochemical, stimulus within the muscle. This is a new area of study that promises important findings in the sphere of molecular and cellular mechanisms involved in the relationship between oxidative stress and exercise.
\end{abstract}

\section{Introduction}

In recent years, there has been major progress in the redox biology of exercise making this review paper highly relevant to the area of Sport Science. Studies have shown that physical exercise increases the production of reactive species and free radicals. Although in excess the unbalance between these oxidant molecules and the body's antioxidants can lead to detrimental effects in our organism, reactive species produced during exercise have an essential role in muscle adaptation to exercise, as studies show.

This review starts by explaining the different modes of exercise, and then offers an overview of the exerciseinduced production of reactive species. This is followed by a description of the body's antioxidant network, and specific details of enzymatic and nonenzymatic antioxidants are given. The efficacy of dietary antioxidant supplementation is also discussed. Finally, the review presents important findings on how reactive species during exercise lead to favourable exercise-induced adaptations, such as enhancement in the antioxidant enzyme activity, angiogenesis, mitochondria biogenesis, and muscle hypertrophy. Our approach provides a general understanding of the subject and presents interesting new findings; when necessary, reference is made to more specific reviews.

\section{Definition of Exercise}

Exercise can be defined as any planned structured activity that leads to increase in energy expenditure and heart rate. There are different modes of exercise in relation to intensity (aerobic and anaerobic), to muscle contraction (isometric, 
concentric, and eccentric), and to frequency (acute and chronic). It is necessary to explain each of these modes of exercise so the reader can better understand the influence of exercise in reactive species production and its consequence in angiogenesis, hypertrophy, and mitochondria biogenesis.

2.1. Differences between Aerobic and Anaerobic Exercise. The main physiological difference between aerobic and anaerobic exercise is the energy source. On one hand, aerobic exercise can be characterized by the use of aerobic metabolism during the physical effort. In this case, the aerobic metabolism primarily generates energy from fat, and with the use of oxygen it produces energy, without much accumulation of lactic acid in the blood. On the other hand, anaerobic exercise is characterized by short periods of high to maximal efforts when energy is supplied via the anaerobic metabolism, that is, without the use of oxygen, and this results in high accumulation of lactic acid in the blood [1,2]. Examples of aerobic-endurance exercises are jogging, running, swimming, rowing, and cycling when performed around 50-75\% of participants' maximal aerobic capacity $\left(\mathrm{VO}_{2 \max }\right)$ and for relatively prolonged periods of time. Examples of anaerobic exercises are sprints (while swimming, biking, or running), long jumps, competitive weightlifting, or anything lasting up to 2 minutes and performed above the participants' lactate threshold (e.g., $>75 \% \mathrm{VO}_{2 \max }$ for trained individuals).

2.2. Differences between Isometric, Concentric, and Eccentric Exercise. Muscle contraction occurs when the central nervous system transmits a signal to the muscle fibre. Providing that there is enough energy and calcium availability, the muscle fibres generate tension and the muscle may shorten, lengthen, or remain with the same size. Concentric contraction refers to the shortening of the muscle with the production of force. Examples of a concentric exercise includes the curling phase of a situp, where the two ends (origin and insertion) of abdominal muscles are moving closer together, contracting and shortening [1,3]. Eccentric contraction corresponds to the "stretching" of the muscle from a concentric or static position while tensioned. An example of an eccentric exercise is the down phase of a biceps curl, where the opposite force generated by the dumbbell produces a force greater or equal to the one produced to elongate the two ends of the biceps to the initial position [1]. An isometric exercise consists in a muscle contraction taking place without motion in the affected joints, such as when an athlete holds a weight bell static at 90 degree in a biceps curl exercise.

2.3. Differences between Chronic and Acute Exercise. Acute exercise can be characterised by single bouts of exercise that produce temporary metabolic and cardiovascular responses lasting from few minutes up to several hours. Chronic exercise is when exercise bouts are repeatedly performed inducing a training response, where physiological and metabolic adaptations become more visible and long lasting [4]. Both aerobic and anaerobic exercises may be performed either chronically or acutely.
2.4. Components of Fitness. Components of fitness include the duration, the frequency, the intensity, and the type of activity of a training programme; these variables need to be considered carefully to certify that the aims of the programme are successfully attained. It is important to notice that the training strategies to improve, for example, aerobic performance are different from the ones required for strength fitness improvements, and each activity has specific needs that will influence the use of particular fitness components [1]. The type of sport or activity will dictate which kind of exercise should be incorporated to ensure a perfect improvement in physiological performance. Training aerobically is highly recommended for the development of long-distance running performance, but the same training plan does not apply for competitive weightlifting. Therefore, it is important to define the type of sport before applying the components of fitness in training.

It is also crucial to train at a frequency that will provide a stimulus which brings positive physiological adaptations, and balance it with enough recovery time in order to improve performance. Some activities will require higher frequency than others, compensated with adjustments on the intensity of exercise. Intensity is determined by the training target and the current fitness level of the athlete. This is manipulated, for example, by working at a different percentage from their maximal aerobic capacity $\left(\% \mathrm{VO}_{2 \max }\right)$ or repetition maximum (1RM), normally in a progressive way, while the time (duration) of the training session should be controlled in relation to the intensity $[1,3]$. Increasing the duration of an exercise session influences the component of fitness and, when well administered, can lead to positive gains inamongst other aspects-performance.

Different sports and activities will use different energy systems and will recruit different muscle fibres, demanding therefore different training strategies by manipulating the components of fitness. Sprint runners will require higher anaerobic capacity and exercise intensity as well as less exercise bouts compared to long-distance runners. In addition, sports, such as tennis, involve eccentric movements on the lower body, so exercise prescription for that should aim at improving eccentric strength by using similar movements and intensity as the game. It is, therefore, clear that the components of fitness are interconnected and the manipulation of all of them, in a combined manner, is necessary for an improvement in physiological performance [1].

In this review, the effects of aerobic and anaerobic exercise when performed acutely or chronically will be discussed in depth, as will the differences between reactive species production in exercises that use different muscle contraction.

\section{Production of Reactive Species during Exercise}

3.1. Reactive Species and Free Radicals. Reactive species and free radicals are molecules that, due to their molecular instability (e.g., unpaired electron), promote oxidation reactions with other molecules, such as proteins, lipids, and 
DNA, in order to become stabilized [5-7]. Many reactive species are oxygen centred $\left(\mathrm{O}_{3}, \mathrm{H}_{2} \mathrm{O}_{2}\right.$, etc. $)$ and are, thus, denominated reactive oxygen species (ROS). Some ROS are also free radicals, such as superoxide anion $\left(\mathrm{O}_{2}{ }^{-}\right)$and nitric oxide (NO), because they have an unpaired electron [6]. Free radicals and reactive species are essential to our wellbeing, having various regulatory roles in cells. For example, ROS are produced by immune cells-neutrophils and macrophages-during the process of respiratory burst in order to eliminate antigens [8]. They also serve as stimulating signals of several genes which encode transcription factors, differentiation, and development as well as stimulating cellcell adhesion, cell signaling, involvement in vasoregulation and fibroblast proliferation, and increased expression of antioxidant enzymes $[9,10]$. The latter example is observed when individuals perform chronic exercise and will be further discussed in Section 4.

Our body has an elaborate network of antioxidants that acts as a defence system neutralizing free radicals and reactive species. This process allows for the maintenance of homeostasis. Nevertheless, oxidative stress and impaired cellular function may occur if there is an exacerbated increase in the body's oxidant concentrations, overwhelming the available antioxidants. It may also occur if there is depletion in the available antioxidants due to disease or poor diet [11]. In fact, chronic oxidative stress has been suggested as being the cause or consequence of many acute and chronic human diseases $[7,12,13]$, for example, obesity, cardiovascular diseases, cancer, acute lung injury, and multiple sclerosis.

Directly measuring the production of free radicals and reactive species is very difficult due to their high reactivity and low steady-state concentration. Nevertheless, the electron spin resonance (ESR) technique is a direct method of detection of species that have an unpaired electron, generally meaning that it is a free radical [14]. However, this technique, and a couple of other direct measurements, require very expensive equipment and have complicated methods. Thus, for the assessment of oxidative stress indirect methods are mainly used. For the measurement of oxidative stress biomarkers in human muscle tissue, expired air, urine, blood, and nasal lavage can be used [15-19]. According to Powers and Jackson [10], reliable markers of this process need to have the following characteristics: be chemically unique, be chemically detectable and have relatively long half-lives, increase or decrease during the oxidative stress process, and not be affected by other cell cycles in order to avoid confounding factors. In addition, oxidative stress is also commonly measured by analyzing the shift in the body's antioxidant system and the activity of specific antioxidant enzymes [5].

3.2. Exercise-Induced Oxidative Stress. Under basal conditions the skeletal muscle produces superoxide anions and NO at a low rate. However, during contractile activity, this rate is drastically increased. In fact, aerobic exercise is associated with an increase in oxygen uptake both by the whole body and especially by the contracting muscle group. Sen [20] reported an increase of 10-15-fold in the rate of whole body oxygen consumption and an increase of more than 100fold in the oxygen flux in active muscles during whole-body aerobic exercise.

Although the direct evidence for ROS production during exercise is limited $[14,21,22]$, mainly due to limitations of the methods used, there is an abundance of the literature providing indirect support that oxidative stress might occur during aerobic and anaerobic exercise (for review on exercise-induced oxidative stress, see Fisher-Wellman and Bloomer [5]). When analysing the available literature, there are some different findings regarding the oxidativestress rates as the result of exercise performance. This is comprehensible because a variety of factors can influence the oxidative rate, such as the muscle groups recruited, the modes of contraction, the exercise intensity, the exercise duration, and the exercising population. Therefore, the exercise models used in studies deserve critical evaluation.

\subsection{Mechanisms of Increased Free-Radical Production with Exercise. The causes of increased free radical and ROS production during exercise have not been totally clarified. Although various mechanisms have been identified, there is still a lack of understanding of how each one of them contributes to the total amount of oxidative stress produced. In addition, these mechanisms may act synergistically, and different types of exercise probably elicit different pathways of free radical production [23]. For instance, although the general consensus is that, during exercise, reactive species production occurs mainly by contracting muscle (skeletal and heart), other mechanisms, such as, inflammatory pro- cesses and increased release of catecholamine, that may occur with exercise, also play an important role in the generation of reactive species. The main mechanisms for reactive species production are described below.}

Electron Leak at the Mitochondrial Electron Transport Chain. This theory has led to the interpretation that a substantial increased free radical generation is to be expected during exercise due to a "leak" of electrons in the respiratory chain in the mitochondrial inner membrane of the contracting muscle cells. This would occur due to the inadequate coupling of the electron transfer between the complexes I and III (Figure 1) [23, 24]. It seems that complex Inamely, the iron-sulfur clusters-releases the reactive oxygen species - superoxide anion-only towards the mitochondrial matrix, whereas complex III-the ubiquinol oxidation sitereleases superoxide into both matrix and outside the inner membrane $[24,25]$. Despite this theoretical appeal, there is little direct evidence that mitochondrial superoxide anion production is increased during exercise. In addition, there are studies with isometric exercise where the oxygen pressure $\left(\mathrm{PO}_{2}\right)$ in the mitochondria was proven to be low but still demonstrated an increase in the oxidative stress [26]. Bailey et al. [21] also demonstrated that free radical outflow of a contracting muscle was associated with decreases in intracellular $\mathrm{PO}_{2}$ rather than with conditions of increased oxygen flux. These studies reinforce the fact that it is unlikely that an increase in mitochondrial oxygen flux is the only, 


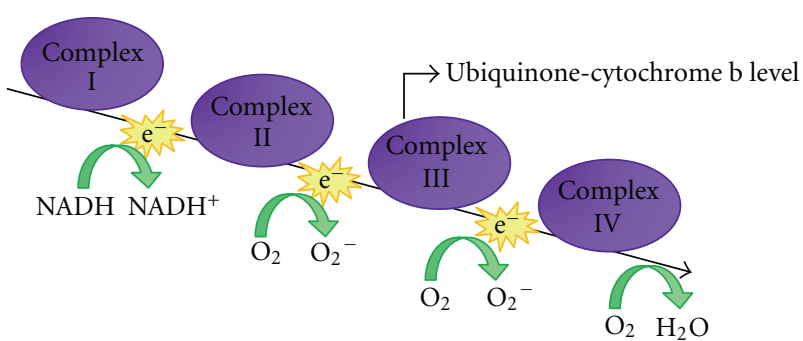

FIgURE 1: The mitochondrial respiratory chain. Electrons are transferred from complexes I, II, and III to IV. However, inadequate coupling of electron transfer can cause leakage, generating superoxide anions at different complex levels.

or main, cause for increases in radical production during exercise [23].

Ischemia Reperfusion and Activation of Endothelial Xanthine Oxidase. The enzyme xanthine oxidase is known to be involved in the pathophysiology of the ischemia-reperfusion syndrome and can lead to tissue damage that may occur after an exhaustive bout of exercise [27]. The following mechanisms describe this process. During exercise, blood flow is shunted from many organs and tissues and redirected to the working muscles; this ischemic condition triggers the conversion of the enzyme xanthine dehydrogenase to xanthine oxidase; when the exercise ceases and the tissues are reoxygenized, xanthine oxidase produces superoxide $\left(\mathrm{O}_{2}{ }^{-}\right)$ and $\mathrm{H}_{2} \mathrm{O}_{2}$ as byproducts of the degradation of hypoxanthine into xanthine and subsequently into uric acid [7], Figure 2. Although this has been shown to happen in few studies, more research is necessary to determine the role that endothelial xanthine oxidase plays in exercise-induced ROS production in humans.

Neutrophils and the Inflammatory Response. As a consequence of an exercise bout, tissue damage or an increase in the inflammatory cell pool may lead to a rise in reactive species production from nonmuscle source. When neutrophils or other phagocytic cells are activated, they release ROS. Despite the fact that this inflammatory response is critical to the removal of damaged proteins and infections, ROS and other oxidants released from these cells can also cause secondary damage, such as lipid peroxidation. Exercise can elicit muscle injury accompanied by the activation of neutrophils [28]. Bøyum et al. [29] showed not only an increase in neutrophil number following aerobic exercise (cycling for $65 \mathrm{~min}$ at $75 \% \mathrm{VO}_{2 \max }$ ) but also an increase in their respiratory burst activity measured as chemiluminescence. An increase in neutrophil counts has also been described in short duration (less than $20 \mathrm{~min}$ ) resistance exercise [30]. This reinforces the fact that there is an increase in plasma neutrophils even when oxygen consumption during physical activity is only moderately increased.

NADPH Oxidase Structure. The reduced form of nicotinamide adenine dinucleotide phosphate (NADPH) oxidase

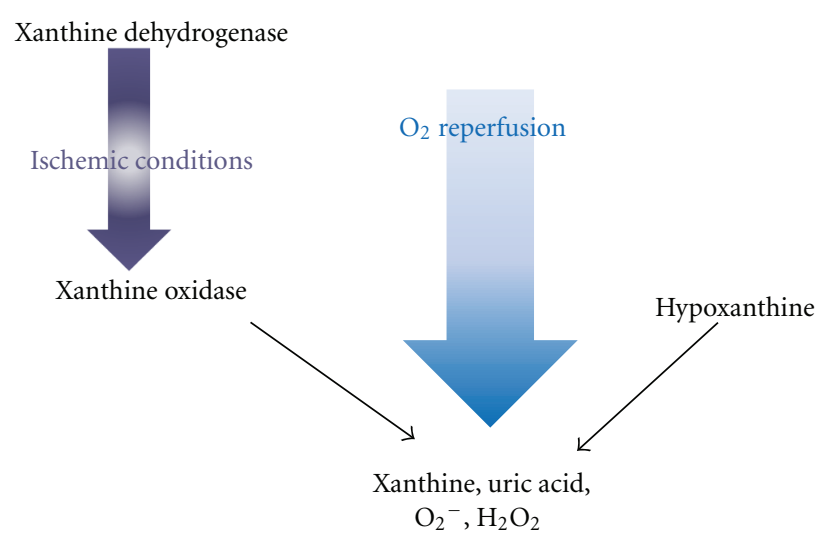

Figure 2: A suggested mechanism for the production of free radicals upon reoxygenation of ischemic or hypoxic tissues.

complex was originally identified and characterized in phagocytes, where it plays an essential role in nonspecific host defense against microbial organisms [31]. Nevertheless, this complex is also found at many sites in skeletal muscle, cellular endothelium, and plasma membrane [10]. NADPH oxidase is normally quiescent, but, when it becomes activated, during muscle contraction or when recruited for antimicrobial and proinflammatory events, it can generate large amounts of $\mathrm{O}_{2}{ }^{--}$that can be converted in $\mathrm{H}_{2} \mathrm{O}_{2}$ by the antioxidant superoxide dismutase [31].

Autooxidation of Catecholamines. Adrenaline, noradrenaline, and dopamine are often referred to collectively as catecholamines. With exercise, there is an increase in the plasma concentration of these substances [32]. The oxidation of catecholamines can produce superoxide anion, $\mathrm{H}_{2} \mathrm{O}_{2}$, and other nonoxygen-derived species in a complicated series of reactions. This can lead to a depletion of cellular antioxidant concentration in the blood, such as glutathione, altering the redox (oxidation-reduction) balance $[7,10]$.

Despite the need for a better comprehension of how these mechanisms of ROS generation interact, the mechanism by which they affect the function of exercising muscles is well established. At rest, the muscles' low concentration of ROS is critical for force generation. During muscle contractile activity there is a rise in ROS production. On one hand, production of reactive species during both aerobic and anaerobic nonexhaustive exercise has been shown to be important for the adaptation of the muscle fibres [33] this process will be discussed in Section 5. Nevertheless, during strenuous exercise, the production of ROS can be higher than the buffering capacity of the antioxidants of the muscles. As ROS accumulates in the contracting muscles, the oxidation of proteins and lipids might cause, amongst other things, inhibition in force production, contributing to the development of acute fatigue [10,34]. In addition, this exaggerated increase in ROS levels in response to strenuous exercise can also lead to oxidative DNA modification, inhibit locomotory and bactericidal activity of neutrophils, reduce the proliferation of $\mathrm{T}$ lymphocytes and $\mathrm{B}$ lymphocytes, 
inhibit natural killer cells, damage cell membrane, and other cellular compounds [35, 36].

\section{The Antioxidant System and Exercise}

Due to the potential role that reactive species and free radicals have in lipid, protein, and DNA damage, it is not surprising that a network of antioxidant defense mechanism is present in the body. In general, antioxidants are often reducing agents, which exist both intracellularly and extracellularly and have the capacity to react with free radicals and reactive species, minimizing their actions and, thus, delaying or preventing oxidative stress [10].

Antioxidants can be both synthesized in vivo and absorbed through diet. They can be divided into two groups: enzymatic and nonenzymatic. The main enzymatic antioxidants include superoxide dismutase (SOD), glutathione peroxidase (GPX), and catalase (CAT). Each of these enzymes is responsible for the reduction of a different ROS, and they are located in different cellular compartments. (1) SOD: there are 3 isoforms of this antioxidant, two of them are present within cells, whereas the other one is located in the extracellular space. Specifically in skeletal muscle cells, the highest percentage of SOD (65-85\%) is found in the cytosol, and the remaining $(15-35 \%)$ is present in the mitochondria of the muscles. SOD catalyses the reaction of superoxide radicals into oxygen and hydrogen peroxides $\left(\mathrm{H}_{2} \mathrm{O}_{2}\right)$. (2) GPX: located in both the cytosol and the mitochondria of cells, it is responsible for the removal of a wide range of hydroperoxides-from complex organic hydroperoxides to $\mathrm{H}_{2} \mathrm{O}_{2}$ - thus, it may protect membrane lipids, proteins, and nucleic acids from oxidation. GPX is also present in muscle cells, but its activity varies depending on the muscle fibre type, with the greatest activity present in slow twitch muscle fibres (type I) which have higher oxidative capacity. (3) CAT: it is extensively distributed within the cells, and its main function is to degrade $\mathrm{H}_{2} \mathrm{O}_{2}$ into $\mathrm{H}_{2} \mathrm{O}$ and $\mathrm{O}_{2}$. Nevertheless, it has a lower affinity for $\mathrm{H}_{2} \mathrm{O}_{2}$ compared with GPX. Similarly to the latter, CAT can be found in higher concentration in type I muscle fibres (for further details on these enzymes, refer to Powers and Jackson [10]).

The nonenzymatic antioxidant group includes glutathione, vitamin $\mathrm{C}$, vitamin $\mathrm{E}$, carotenoids, uric acid, and others. Similarly to the enzymatic antioxidants, these are present in different cellular compartments and elicit distinct antioxidant properties which maximize their effectiveness [11]. Below are more details on the nonenzymatic antioxidants - glutathione, vitamin C, and vitamin E.

Glutathione. Reduced glutathione (GSH) is a water-soluble low-molecular-weight tripeptide formed from the amino acids glutamate, cysteine, and glycine. These three amino acids can be obtained from food intake. All types of cells are capable of synthesizing GSH. This synthesis process occurs through two sequential reactions and requires the action of two enzymes: for the first reaction, $\gamma$-glutamylcysteine and GSH synthetases for the consecutive reaction (Figure 3). Levels of the produced GSH act as a feedback for the control

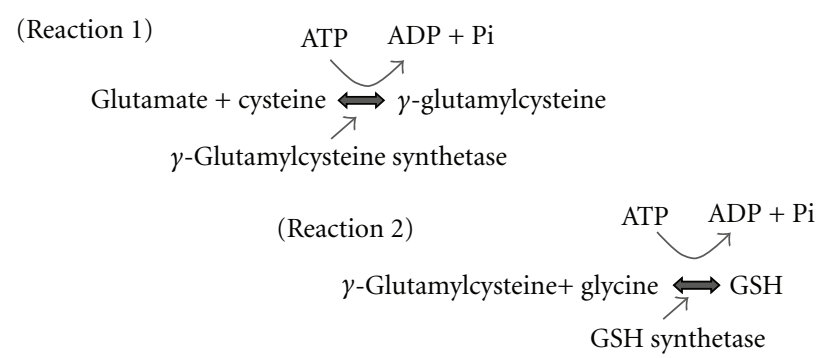

FIGURE 3: GSH synthesis illustrated by two reactions. Enzymes that catalyze the reactions are $\gamma$-glutamylcysteine synthetase and GSH sythetase.

of reaction 1 (see Figure 3), this helps maintain its adequate production [37]. GSH can also be obtained directly from food, but only small amounts of the intact tripeptide are absorbed by the gut; most are broken down into amino acids, which are then used in the synthesis cycle. Thus, a healthy diet with a balanced protein intake is essential for an adequate GSH homeostasis in the body $[38,39]$.

GSH exerts various essential functions in the body. Amongst these functions is its major antioxidant role. It efficiently scavenges ROS and free radicals preventing an increase in the oxidative stress process. In these reactions, the reduced GSH is oxidized, via the enzyme glutathione peroxidase, to form glutathione disulfide (GSSG). Note that GSSG is formed by two GSH molecules linked via a disulfide bond due to oxidation of the thiol $(\mathrm{SH})$ groups. Once oxidised, GSSG can be reduced back to its original GSH form by the enzyme GSSG reductase and nicotinamide adenine dinucleotide phosphate (NADPH). Nevertheless, when there is a high level of oxidative stress, NADPH becomes depleted and there is an intracellular accumulation of GSSG. This excess GSSG can either be exported out of the cell or it can form a mixed disulfide. Despite what happens to the oxidized glutathione, depletion of cellular GSH can be observed when an intense oxidative stress process occurs [40].

Measuring the plasma level of GSH or its oxidized form (GSSG) is a widely accepted method of detecting oxidative stress and can be reported as redox potential, GSH or GSSG concentration, or GSH/GSSG ratio. It is not only a good indicator of systemic oxidative status but also a useful indicator of disease risk [41], and, therefore, it has been used in various studies to indicate the free radical production during exercise [19, 42-44]. However, these studies do present some divergent results which could possibly be explained by the difference in subject fitness, exercise protocol, and method of determining the glutathione concentration.

Vitamin C. It is a water-soluble vitamin and refers to both ascorbic acid and dehydroascorbic acid (DHA). Ascorbic acid is the main form of the vitamin found in vivo. This vitamin, also referred to as ascorbate, is found in relatively high levels in different tissues throughout the body. Ascorbate has clearly been shown to play an essential role in connective tissue biosynthesis, and its deficiency results in scurvy, a disease which leads to the deterioration of collagen 
production and results in fragile blood vessels and impaired lesion healing. This disease is reversible once the individual restarts the ingestion of ascorbate. Vitamin $\mathrm{C}$ is also a strong reducing agent, due to its facility in donating electrons, with important antioxidant properties [45]. It can inactivate a variety of reactive species minimizing damage to body tissues.

During oxidation reactions, only small amounts of ascorbate are lost because, once it is oxidized, it can be reduced back to ascorbic acid by reductants such as glutathione, nicotinamide adenine dinucleotide (NADH), and NADPH. Similarly, vitamin $\mathrm{C}$ is also known to regenerate other antioxidants, such as vitamin $\mathrm{E}$ and glutathione, back to their reducing state; thus, maintaining a balanced network of antioxidants [46].

When ingested, vitamin $\mathrm{C}$ is absorbed in the intestines either by active transport, if the availability of the vitamin is low, or by simple diffusion if it is present in high concentrations. If ingested in excess, it is degraded in the intestines and can cause diarrhea and intestinal discomfort. Besides the absorption and degrading mechanisms occurring in the intestines, the kidneys are also responsible for conserving or eliminating unmetabolized ascorbic acid to help maintain the body's ascorbic acid balance [45].

Vitamin E. Is a lipid-soluble vitamin also referred to as $\alpha$ tocopherol. There are a variety of vitamin E molecules that differ in structure. The various forms of this vitamin differ significantly in their metabolic functions and bioavailability. In humans, over $90 \%$ of vitamin $\mathrm{E}$ encountered in the body is $\alpha$-tocopherol. However, not all the $\alpha$-tocopherol forms are maintained in the plasma. The $\alpha$-tocopherol, which is encountered naturally in food together with synthetic forms of the $\alpha$-tocopherol (the isomers RRR-, RSR, RRS-, and RSS- $\alpha$-tocopherol), can be maintained in the human plasma and tissues. When ingesting $\alpha$-tocopherol supplements, the RRR- $\alpha$-tocopherol form is preferable $[45,47]$.

This vitamin has been shown to have beneficial effects in relation to some diseases, it has also been associated with a decreased risk in cardiovascular disease, and it can help slow the progress of degenerative diseases, such as atherosclerosis. In contrast, in patients suffering from impairment in intestinal fat absorption, $\alpha$-tocopherol deficiency has been associated with neuronal degeneration [48]. Nevertheless, a recent review study [49], which thoroughly analyzes over 47 antioxidant supplementation research papers on allcause mortality, has unexpectedly shown the negative effect of some supplements. Vitamin E, for example was one of the antioxidants that was associated with an increase in mortality, while Vitamin $\mathrm{C}$ did not show a similar effect. It is difficult to determine the specific biochemical and physiological mechanisms that may have led to this result. More investigation is, therefore, necessary to answer questions that such research generates. In addition, these results concerning synthetic antioxidants should not be transferred to effects that fruit and vegetables have on human health.
Similarly to vitamin C, vitamin E has important antioxidant properties. Due to its capacity for scavenging ROS and free radicals, particularly peroxyl radical $\left(\mathrm{ROO}^{\bullet}\right)$, it exerts the important function of protecting cellular membranes and plasma lipoproteins against lipid peroxidation. This is possible because vitamin $\mathrm{E}$ has a great affinity for reducing peroxyl radicals, preventing their interaction with the membrane phospholipids or lipoproteins [45]. Indeed, vitamin E can be considered one of the major inhibitors of lipid peroxidation in vivo [7]. Once oxidized, it can be regenerated back to its reduced state by vitamin C, as mentioned previously. Nevertheless, research shows that increased levels of $\alpha$-tocopherol radicals that are not converted back to the reduced form can act as prooxidants, initiating oxidative stress processes by themselves. In addition, an environment that lacks other antioxidants or that has a high level of oxidative stress will contribute to vitamin E acting as a prooxidant [46].

The evidence that exercise leads to an increase in free radicals and reactive species has led researchers to analyse the efficacy of dietary antioxidant supplementation in order to attenuate the muscle oxidative stress generation and thus improve muscular performance and immune function. An example that can be mentioned is the supplementation with vitamin E. As mentioned at the start of this section, vitamin $\mathrm{E}$ has been found to protect cellular membranes from lipid peroxidation. Hence, it is logical to assume that this vitamin could protect muscle cells against exerciseinduced damage. Early studies, analyzing the effects of vitamin E supplementation and exercise, investigated its effect on performance. Most of the studies, however, report no benefit of vitamin $\mathrm{E}$ neither for muscle strength nor for endurance performance [50]. Furthermore, it has been hypothesized that vitamin E supplementation could have a protective effect against the contraction-induced muscle damage oxidative stress that may occur after an intense exercise bout. This rationale is based on the knowledge that this vitamin can stabilize muscle membranes by interacting with its phospholipids which would, this way, provide some protection against the increase in oxidative stress or muscle damage observed after certain types of exercise [50]. Yet results of the various studies have been quite contradictory, and it is not within the scope of this review to discuss these issues in depth, for further details refer to the review of Jackson et al. [51].

Interestingly, recent work has shown that the increase in reactive species during exercise leads to favourable exerciseinduced adaptations. Pertaining literature documents well that both aerobic and anaerobic training causes an enhancement in the antioxidant enzyme activity in various tissues $[33,52,53]$. This is an adaptation process that happens because the free radicals, produced during muscle contraction, act as signalling molecules. This stimulates the gene expression and, hence, increases production of antioxidant enzymes and modulates other oxidative stress protection pathways, such as enhancing the activity of DNA repair enzymes in skeletal muscles [33, 54]. This strengthens the body's antioxidant network system which, consequently, minimizes the oxidative stress process [33]. This stimuli 
associated with enhanced antioxidant protection occurs not only in the muscles but also systemically so vital organs, such as liver and brain, also go through this beneficial adjustment [55].

This adaptation process, resultant from regular exercise stimulus, can be explained by the hormesis theory. This theory postulates that chemicals and toxic substances may have a low-dose stimulation high-dose inhibitory effect. That means they can provide positive responses when present in small amount [56]. In this regard, the type and duration of training are key for a significant upregulation of the endogenous antioxidants with long-duration high-intensity endurance training being more effective [57]. Niess et al. [58] reported that trained individuals presented less DNA damage after an exhaustive bout of exercise compared to untrained men. Other evidence for this training adaptation was reported by Miyazaki et al. [59], which showed that free radical production was reduced after 12 weeks of endurance training. More specifically, they reported a decrease in the neutrophil superoxide anion production and attenuation in the lipid peroxidation process. The contrary is also true as sedentarism not only reduces various physiological functions but also decreases the body's oxidative stress protection mechanisms. Consequently, there may be an increase in the occurrence of oxidative stress associated with diseases such as cancer, atherosclerosis, cardiovascular, and neurodegenerative diseases [55].

\section{Does ROS Play an Important Role in Exercise-Induced Adaptation Response?}

As previously mentioned, oxidative stress is often used to indicate a condition in which accumulation of reactive species has damaging effects in many tissues and organs [7]. However, emerging pieces of evidence have shown that oxidative stress plays a critical role in muscle homeostasis and exercise metabolism of skeletal muscle. This suggests that reactive species are not merely damaging agents inflicting random destruction to cell structures and functions, but that they are also-at least within physiological concentrationuseful signalling molecules that regulate growth, proliferation, differentiation, and may be responsible for some adaptations in exercised tissue and in nonexercised tissues, such as the brain $[55,60]$. Therefore, physical exercise induces acute increased production of reactive species in skeletal muscle, which can work as intracellular signaling molecules [61, 62]. But, how do reactive species act as physiological signaling molecules in exercise-induced remodeling tissue and who are the most important players in this game?

5.1. Angiogenesis. Angiogenesis is the process through which activated endothelial cells branch out from an existing capillary [63]. The growth of new blood vessels is an important natural process required for healing wounds and for restoring blood flow to tissues after injury or insult [64]. It is known that endurance training induces angiogenesis responses on muscle tissue [63]. For endurance athletes, this adaptation is very important due to the increased need of oxygen supply to all muscle mass. The process of angiogenesis is controlled by a number of mediators that are released in the tissues surrounding the small vessels [64]. Physical exercise can activate this process contributing with the release of some mediators. Significant variation (physiological or physiopathological) in mechanical forces that occur in vivo cannot be countered by acute regulation of vessel wall diameter, leading to phenotypical modulation of the endothelial cells and vascular cells, producing structural modifications of the arterial wall. As such, vascular remodeling is a fundamental basis of normal vessel growth and exercise-induced adaptation.

Recently, the role of ROS in high flow-induced vascular remodeling was demonstrated in a mouse model of arteryjugular vein (AVF) where ROS production was enhanced in arteries exposed to chronic high flow, both 1 and 3 weeks after opening of the AVF [65]. NADPH oxidase is identified as the major generator of shear stress-induced ROS in the AVF vascular wall. Generation of ROS and NO, derived from endothelial oxide nitric synthase (eNOS) activation, led to matrix metalloproteinases (MMP) activation, in AVF, and modulated flow-induced vascular enlargement. MMPs collectively cleave most, if not all, of the constituents of the extracellular matrix. Observations made in $\mathrm{p} 47 \mathrm{phox}^{-/-}$ mice and $\mathrm{eNOS}^{-/-}$mice also provided direct evidence that endogenous ROS and NO modulated not only the activity of MMPs but also their production in arteries exposed to elevated blood flow. Lehoux [66] showed that long-term structural adaptation to altered blood flow is mediated by ROS. In addition, Lehoux et al. [67] demonstrated that high intraluminal pressure-induced MMP-9 in carotid arteries contributed to increased vessel distensibility. This factor points to the role of matrix degrading enzymes in the early stages of vascular remodeling.

As is well known, the key to angiogenesis is the vascular endothelial growth factor (VEGF), but the real relationship between exercise and angiogenesis is not completely understood. Initial studies on this topic were conducted in skeletal muscle. An upregulation of VEGF, following chronic muscle stimulation or a single bout of moderately intense treadmill running, occurs in the skeletal muscle of rats [68]. In the case of treadmill running VEGF mRNA was upregulated in the active muscle of rats approximately 2 - to 4 -fold by the end of the exercise bout and remained elevated for the following $4 \mathrm{~h}$. Eight hours after exercise, the VEGF mRNA levels had returned to that of resting muscle.

According to Gustafsson et al. [69], upregulation of VEGF mRNA also occurs during exercise in healthy humans. Similarly, exercise upregulates other elements that are important in the angiogenesis process, for example, the mRNA of the VEGF receptors 1 and 2 (VEGFR1 and VEGFR2 resp.) are increased after muscle activity [70]. Therefore, it seems that aerobic exercise can amplify the angiogenic cascade.

Another important and recently investigated player in the angiogenesis exercise-induced process is PGC- $1 \alpha$ (peroxisome-proliferator-activated receptor-c coactivator$1 \alpha$ ). Arany et al. [71] have shown that the PGC- $1 \alpha$ is a potent metabolic sensor and regulator, induced by a lack of nutrients and oxygen. In addition, PGC- $1 \alpha$ powerfully 
regulates VEGF expression and angiogenesis in cultured muscle cells and skeletal muscle in vivo. Exercise can induce a temporary ischemia in muscular tissue [72]. Ischemia leads to a profound metabolic challenge with potentially catastrophic consequences. For example, PGC- $1 \alpha^{-/-}$mice showed a striking failure to reconstitute blood flow to the limb, in a normal manner, after an ischemic insult, whereas transgenic expression of PGC- $1 \alpha$ in skeletal muscle is protective.

West et al. [73] described a novel mechanism of angiogenesis that is independent of hypoxia-triggered VEGF expression. The products of lipid peroxidation are generated as a consequence of oxidative stress and are recognized by toll-like receptors (TLR) promoting angiogenesis in vivo, thereby, contributing to accelerate wound healing and tissue recovery. Capillarity in active skeletal muscle is significantly increased by endurance exercise training, and, according to Bloor [63], any increase in muscle capillarity is important in improving blood-tissue exchange properties. This is because a greater capillary network would (1) increase the surface area for diffusion, (2) shorten the average diffusion path length within the muscle, and (3) increase the length of time for diffusive exchange between blood and tissue. Therefore, the persistence of newly formed vasculature in an inflammatory context leads to the maintenance of tissue remodeling. More studies are necessary to define the relationship of immune cells and other molecules and receptors, such as VEGF, PGC1- $\alpha$, and TLR2, during the neovasculature process in response to exercise. This would be of relevance for creating training strategies for optimal tissue repair.

5.2. Mitochondrial Biogenesis. Regular aerobic training produces an adaptation in skeletal muscle termed mitochondrial biogenesis [74], that is, increase in the number and size of mitochondria [75]. The mechanism behind this adaptation is not clear, but recent studies suggest a possible role for ROS in this process. Although considerable focus has been placed on the damage created by production of ROS, it is also known that ROS can activate signaling pathways involved in phenotypic adaptations. Researches demonstrate that ROS induce mitochondrial network branching and elongation. Pesce et al. [76] showed that mtDNA copy number increased with rising levels of ROS in aging skeletal muscle, and this increase, in mtDNA, was accompanied by an induction in mitochondrial mass. This response appeared to be mediated by PGC- $1 \alpha$ and nuclear respiratory factor 1 (NRF-1) since the expression of both increased following exogenous ROS treatment [77]. Recently, Irrcher et al. [78] demonstrated that ROS can lead to an increase in PGC- $1 \alpha$ promoter activity and expression via both adenosine monophosphateactivated protein kinase-dependent (AMPK) and AMPKindependent pathways. These pathways most likely account, in part, for the increase in mitochondrial biogenesis observed in the presence of ROS.

5.3. Skeletal Muscle Hypertrophy. The literature suggests that reactive species have important roles in the regulation of cell signaling and changes in gene expression [79, 80], contributing, amongst other processes, to the control of skeletal muscle size. In vitro evidence indicates that ROS are capable of affecting the efficiency of muscular tissue differentiation and successful differentiation of satellitederived myoblasts (progenitor cells that originate muscle cells) into functioning. Integrated myotubes is a fundamental prerequisite for muscle regeneration, a repair process which is of primary importance in maintaining muscle function [61].

ROS modulate the signaling of various growth factors via redox regulation. However, the role of ROS in insulin-like growth factor-1 (IGF-1) signaling is not fully understood. IGF-1 is a hormone with a similar molecular structure to insulin. It is a very important player in cell proliferation, differentiation, and survival, performing various tissuespecific functions [81]. IGF-1 induces myocyte (also known as a muscle cell) hypertrophy, particularly in skeletal muscle [82]. In vivo, muscle-specific transgenic mice overexpressing IGF-1 exhibit increased muscle mass, and mice specifically lacking IGF-1 receptor (IGF-IR) in their muscles have smaller muscles and reduced number of myofibers [83]. Hadayaningsih and coworkers [84] recently investigated whether ROS regulate the signaling and biological action of IGF-1 in C2C12 myocytes in mice. They found that IGF-1 induces ROS in C2C12 myocytes; while treatment with $\mathrm{H}_{2} \mathrm{O}_{2}$ significantly enhanced IGF-I-induced phosphorylation of the IGF-1 receptor (IGF-1R), phosphorylation was markedly attenuated when cells were treated with antioxidants. The downstream signaling pathway Akt/mTOR-p70S6K (a wellknown hypertrophic pathway) was subsequently downregulated, and, furthermore, blocking Nox4-which is reported to produce ROS in insulin signaling-attenuated IGF-1induced IGF-1R phosphorylation, indicating that Nox4 is involved in the regulation of IGF-1 signaling. Importantly, antioxidant treatments inhibited IGF-1-induced myocyte hypertrophy, demonstrating that ROS are necessary for inducing IGF-1 myocyte hypertrophy in vitro. These results indicate that reactive species play an essential role in the signaling and the biological action of IGF-1 in C2C12 myocytes, clearly showing that IGF-1 is an essential regulator of myocyte hypertrophy. As strength exercise stimulates skeletal muscle hypertrophy and local IGF-1 expression, this suggests the involvement of IGF-1 in exercise-induced myocyte hypertrophy.

Another molecule that appears to have an important role in the hypertrophic response to specific kind of exercise is interleukin-6 (IL-6). Recent scientific literature identifies IL6 as an essential regulator of satellite cell-(muscle stem cell-) mediated hypertrophic muscle growth. IL-6 is locally and transiently produced by growing myofibres and associated satellite cells, and genetic loss of IL-6 blunted muscle hypertrophy in vivo [85]. Kosmidou et al. [86] have shown that ROS stimulate IL-6 release from skeletal myotubes, therefore, identifying a novel cellular source which could be responsible for the excess amounts of inflammationresponsive cytokine produced in strenuous-exercise models. Their results also suggest that the ROS-stimulated increase in IL-6 release is transcription dependent and involves p38 and 
$\mathrm{NF} \kappa-\mathrm{B}$ activation. Although p38-MAPK emerges as a pivotal molecule orchestrating sequential events in the myogenic pathway, many details of p38-MAPK-induced myogenesis are yet to be elucidated. Whether different p38 MAPK family members specifically regulate the expression of particular subsets of genes, at different stages of differentiation, and whether they possess inducing or repressing activities are still to be determined [87].

A balance between reactive species production and antioxidant defense systems appears to represent a central key of many adaptive responses in skeletal muscle as well as hypertrophy and atrophy. It looks like, if the production of ROS is ideal (little higher than basal levels), the adaptive response is hypertrophy; but if the levels of ROS are increased many folds above basal levels and antioxidant defense capacity, there is an atrophic response such as Duchenne muscular dystrophy [88].

\section{Conclusions}

As has been shown, a sophisticated signaling-transcription network within muscle fibres mediates exercise-induced skeletal muscle adaptation, and there is strong evidence that ROS has important role in this scenario. ROS participates in angiogenesis, mitochondrial biogenesis, and hypertrophy that have an important role in exercise prescription and athletic performance. In relation to acute exercise and free radical production, both anaerobic and aerobic exercise may result in augmented production of free radicals, but acute oxidative stress may not always be seen since ROS production is positively dependant of the load (intensity $\times$ duration) of exercise. It has been suggested that high ROS produced by acute bouts of exercise may be detrimental to the immune system; however, chronic exercise produces physiological adaptations capable of upregulating one's antioxidant system. In this regard, the type and duration of the training are key for a significant upregulation of the endogenous antioxidants with long-duration high-intensity endurance training having been shown to be more effective.

\section{Future Perspectives}

The past 3 decades has brought enormous progress to our understanding of the impact of reactive species on exercise adaptative response. The muscular sources of ROS have been identified, and many of the signaling pathways that are modified by ROS have been studied; however, much remains to be discovered regarding the function of ROS in the specific adaptative response as angiogenesis, hypertrophy, and mitochondrial biogenesis. There is now a better understanding of the regulation of ROS sources during exercise, but that still needs to be further explored. Additional work is necessary to elucidate the specific role of reactive species in migration processes like rolling, adherent, and transmigrating cells; mechanistic adaptation processes need to be investigated. Finally, there are many researchers working to extend our knowledge of the molecular and cellular mechanisms involved in the relationship between oxidative stress and exercise.

\section{References}

[1] D. Lawrence and R. Hope, Advanced Circuit Training: a Complete Guide to Progressive Planning and Instructing, A \& C Black, London, UK, 2008.

[2] W. D. McArdle, F. I. Katch, and V. L. Katch, Exercise Physiology: Nutrition, Energy, and Human Performance, Lippincott Williams \& Wilkins, Philadelphia, Pa, USA, 2009.

[3] P. V. Komi, Strength and Power in Sport, John Wiley \& Sons, Oxford, UK, 2003.

[4] P. D. Thompson, S. F. Crouse, B. Goodpaster, D. Kelley, N. Moyna, and L. Pescatello, "The acute versus the chronic response to exercise," Medicine and Science in Sports and Exercise, vol. 33, no. 6, pp. S438-S445, 2001.

[5] K. Fisher-Wellman and R. J. Bloomer, "Acute exercise and oxidative stress: a 30 year history," Dynamic Medicine, vol. 8, no. 1, pp. 1-25, 2009.

[6] Z. Radák, Free Radicals in Exercise and Aging, Human Kinetics, Champaign, Ill, USA, 2000.

[7] B. Halliwell and J. M. C. Gutteridge, Free Radicals in Biology and Medicine, Oxford University Press, New York, NY, USA, 2007.

[8] M. Freitas, A. Gomes, G. Porto, and E. Fernandes, "Nickel induces oxidative burst, NF- $\kappa \mathrm{B}$ activation and interleukin8 production in human neutrophils," Journal of Biological Inorganic Chemistry, vol. 15, no. 8, pp. 1275-1283, 2010.

[9] C. K. Sen, "Antioxidant and redox regulation of cellular signaling: introduction," Medicine and Science in Sports and Exercise, vol. 33, no. 3, pp. 368-370, 2001.

[10] S. K. Powers and M. J. Jackson, "Exercise-induced oxidative stress: cellular mechanisms and impact on muscle force production," Physiological Reviews, vol. 88, no. 4, pp. 12431276, 2008.

[11] S. K. Powers, K. C. DeRuisseau, J. Quindry, and K. L. Hamilton, "Dietary antioxidants and exercise," Journal of Sports Sciences, vol. 22, no. 1, pp. 81-94, 2004.

[12] C. W. Chow, M. T. H. Abreu, T. Suzuki, and G. P. Downey, "Oxidative stress and acute lung injury," American Journal of Respiratory Cell and Molecular Biology, vol. 29, no. 4, pp. 427431, 2003.

[13] H. Y. Chung, E. K. Lee, Y. J. Choi et al., "Molecular inflammation as an underlying mechanism of the aging process and age-related diseases," Journal of Dental Research, vol. 90, no. 7, pp. 830-840, 2011.

[14] T. Ashton, I. S. Young, J. R. Peters et al., "Electron spin resonance spectroscopy, exercise, and oxidative stress: an ascorbic acid intervention study," Journal of Applied Physiology, vol. 87, no. 6, pp. 2032-2036, 1999.

[15] R. Child, S. Brown, S. Day, A. Donnelly, H. Roper, and J. Saxton, "Changes in indices of antioxidant status, lipid peroxidation and inflammation in human skeletal muscle after eccentric muscle actions," Clinical Science, vol. 96, no. 1, pp. 105-115, 1999.

[16] K. J. Mantione, T. Esch, and G. B. Stefano, "Detection of nitric oxide in exhaled human breath: exercise and resting determinations," Medical Science Monitor, vol. 13, no. 3, pp. MT1-MT5, 2007.

[17] C. M. Shing, J. M. Peake, S. M. Ahern et al., "The effect of consecutive days of exercise on markers of oxidative stress," 
Applied Physiology, Nutrition and Metabolism, vol. 32, no. 4, pp. 677-685, 2007.

[18] O. Neubauer, S. Reichhold, L. Nics et al., "Antioxidant responses to an acute ultra-endurance exercise: impact on DNA stability and indications for an increased need for nutritive antioxidants in the early recovery phase," British Journal of Nutrition, vol. 104, no. 8, pp. 1129-1138, 2010.

[19] E. C. Gomes, V. Stone, and G. Florida-James, "Impact of heat and pollution on oxidative stress and CC16 secretion after 8 km run," European Journal of Applied Physiology, vol. 111, no. 9, pp. 2089-2097, 2011.

[20] C. K. Sen, "Oxidants and antioxidants in exercise," Journal of Applied Physiology, vol. 79, no. 3, pp. 675-686, 1995.

[21] D. M. Bailey, I. S. Young, J. McEneny et al., "Regulation of free radical outflow from an isolated muscle bed in exercising humans," American Journal of Physiology, vol. 287, no. 4, pp. H1689-H1699, 2004.

[22] D. A. Bailey, L. Lawrenson, J. McEneny et al., "Electron paramagnetic spectroscopic evidence of exercise-induced free radical accumulation in human skeletal muscle," Free Radical Research, vol. 41, no. 2, pp. 182-190, 2007.

[23] N. B. J. Vollaard, J. P. Shearman, and C. E. Cooper, "Exerciseinduced oxidative stress: myths, realities and physiological relevance," Sports Medicine, vol. 35, no. 12, pp. 1045-1062, 2005.

[24] X. Xu and E. A. Arriaga, "Qualitative determination of superoxide release at both sides of the mitochondrial inner membrane by capillary electrophoretic analysis of the oxidation products of triphenylphosphonium hydroethidine," Free Radical Biology and Medicine, vol. 46, no. 7, pp. 905-913, 2009.

[25] F. L. Muller, Y. Liu, and H. Van Remmen, "Complex III releases superoxide to both sides of the inner mitochondrial membrane," Journal of Biological Chemistry, vol. 279, no. 47, pp. 49064-49073, 2004.

[26] H. M. Alessio, A. E. Hagerman, B. K. Fulkerson, J. Ambrose, R. E. Rice, and R. L. Wiley, "Generation of reactive oxygen species after exhaustive aerobic and isometric exercise," Medicine and Science in Sports and Exercise, vol. 32, no. 9, pp. 1576-1581, 2000.

[27] M. C. Gómez-Cabrera, F. V. Pallardó, J. Sastre, J. Viña, and L. Garcia-Del-Moral, "Allopurinol and markers of muscle damage among participants in the Tour de France," Journal of the American Medical Association, vol. 289, no. 19, pp. 25032504, 2003.

[28] L. L. Ji, "Antioxidants and oxidative stress in exercise," Proceedings of the Society for Experimental Biology and Medicine, vol. 222, no. 3, pp. 283-292, 1999.

[29] A. Bøyum, O. Rønsen, V. A. Tennfjord et al., "Chemiluminescence response of granulocytes from elite athletes during recovery from one or two intense bouts of exercise," European Journal of Applied Physiology, vol. 88, no. 1-2, pp. 20-28, 2002.

[30] A. Ramel, K. H. Wagner, and I. Elmadfa, "Correlations between plasma noradrenaline concentrations, antioxidants, and neutrophil counts after submaximal resistance exercise in men," British Journal of Sports Medicine, vol. 38, no. 5, p. E22, 2004.

[31] B. M. Babior, J. D. Lambeth, and W. Nauseef, "The neutrophil NADPH oxidase," Archives of Biochemistry and Biophysics, vol. 397, no. 2, pp. 342-344, 2002.

[32] L. V. Ghimire, U. Kohli, C. Li et al., "Catecholamine pathway gene variation is associated with norepinephrine and epinephrine concentrations at rest and after exercise," Pharmacogenet Genomics, vol. 22, no. 4, pp. 254-260, 2012.
[33] M. C. Gomez-Cabrera, E. Domenech, and J. Viña, "Moderate exercise is an antioxidant: upregulation of antioxidant genes by training," Free Radical Biology and Medicine, vol. 44, no. 2, pp. 126-131, 2008.

[34] M. B. Reid, "Nitric oxide, reactive oxygen species, and skeletal muscle contraction," Medicine and Science in Sports and Exercise, vol. 33, no. 3, pp. 371-376, 2001.

[35] C. K. Sen and S. Roy, "Antioxidant regulation of cell adhesion," Medicine and Science in Sports and Exercise, vol. 33, no. 3, pp. 377-381, 2001.

[36] A. M. Niess and P. Simon, "Response and adaptation of skeletal muscle to exercise-the role of reactive oxygen species," Frontiers in Bioscience, vol. 12, pp. 4826-4838, 2007.

[37] M. E. Anderson, "Glutathione: an overview of biosynthesis and modulation," Chemico-Biological Interactions, vol. 111112, pp. 1-14, 1998.

[38] E. Valencia, A. Marin, and G. Hardy, "Glutathionenutritional and pharmacological viewpoints: part III," Nutrition, vol. 17, no. 7-8, pp. 696-697, 2001.

[39] G. Wu, Y. Z. Fang, S. Yang, J. R. Lupton, and N. D. Turner, "Glutathione metabolism and its implications for health," Journal of Nutrition, vol. 134, no. 3, pp. 489-492, 2004.

[40] S. C. Lu, "Regulation of hepatic glutathione synthesis: current concepts and controversies," The FASEB Journal, vol. 13, no. 10, pp. 1169-1183, 1999.

[41] R. Rossi, A. Milzani, I. Dalle-Donne et al., "Blood glutathione disulfide: in vivo factor or in vitro artifact?" Clinical Chemistry, vol. 48, no. 5, pp. 742-753, 2002.

[42] J. Sastre, M. Asensi, E. Gasco et al., "Exhaustive physical exercise causes oxidation of glutathione status in blood: prevention by antioxidant administration," American Journal of Physiology, vol. 263, no. 5, pp. R992-R995, 1992.

[43] C. K. Sen, T. Rankinen, S. Vaisanen, and R. Rauramaa, “Oxidative stress after human exercise: effect of $\mathrm{N}$-acetylcysteine supplementation," Journal of Applied Physiology, vol. 76, no. 6, pp. 2570-2577, 1994.

[44] I. Medved, M. J. Brown, A. R. Bjorksten, J. A. Leppik, S. Sostaric, and M. J. McKenna, "N-acetylcysteine infusion alters blood redox status but not time to fatigue during intense exercise in humans," Journal of Applied Physiology, vol. 94, no. 4, pp. 1572-1582, 2003.

[45] Institute of Medicine, Dietary Intakes for Vitamin C, Vitamin E, Selenium, Carotenoids, National Academy Press, Washigton, DC, USA, 2000.

[46] I. M. C. M. Rietjens, M. G. Boersma, L. D. Haan et al., "The pro-oxidant chemistry of the natural antioxidants vitamin C, vitamin E, carotenoids and flavonoids," Environmental Toxicology and Pharmacology, vol. 11, no. 3-4, pp. 321-333, 2002.

[47] J. K. Lodge, W. L. Hall, Y. M. Jeanes, and A. R. Proteggente, "Physiological factors influencing vitamin E biokinetics," Annals of the New York Academy of Sciences, vol. 1031, pp. 6073, 2004.

[48] F. M. Cerqueira, M. H. G. Medeiros, and O. Augusto, "Antioxidantes dietéticos: controvérsias e perspectivas," Química Nova, vol. 30, no. 2, pp. 441-449, 2007.

[49] G. Bjelakovic, D. Nikolova, L. L. Gluud, R. G. Simonetti, and C. Gluud, "Mortality in randomized trials of antioxidant supplements for primary and secondary prevention: systematic review and meta-analysis," Journal of the American Medical Association, vol. 297, no. 8, pp. 842-857, 2007.

[50] M. L. Urso and P. M. Clarkson, "Oxidative stress, exercise, and antioxidant supplementation," Toxicology, vol. 189, no. 1-2, pp. 41-54, 2003. 
[51] M. J. Jackson, M. Khassaf, A. Vasilaki, F. McArdle, and A. McArdle, "Vitamin E and the oxidative stress of exercise," Annals of the New York Academy of Sciences, vol. 1031, pp. 158168, 2004.

[52] M. Kanter, "Free radicals, exercise and antioxidant supplementation," Proceedings of the Nutrition Society, vol. 57, no. 1, pp. 9-13, 1998.

[53] D. O. Wilson and P. Johnson, "Exercise modulates antioxidant enzyme gene expression in rat myocardium and liver," Journal of Applied Physiology, vol. 88, no. 5, pp. 1791-1796, 2000.

[54] Z. Radák, P. Apor, J. Pucsok et al., "Marathon running alters the DNA base excision repair in human skeletal muscle," Life Sciences, vol. 72, no. 14, pp. 1627-1633, 2003.

[55] Z. Radak, H. Y. Chung, and S. Goto, "Systemic adaptation to oxidative challenge induced by regular exercise," Free Radical Biology and Medicine, vol. 44, no. 2, pp. 153-159, 2008.

[56] Z. Radak, H. Y. Chung, and S. Goto, "Exercise and hormesis: oxidative stress-related adaptation for successful aging," Biogerontology, vol. 6, no. 1, pp. 71-75, 2005.

[57] S. K. Powers, L. L. Ji, and C. Leeuwenburgh, "Exercise traininginduced alterations in skeletal muscle antioxidant capacity: a brief review," Medicine and Science in Sports and Exercise, vol. 31, no. 7, pp. 987-997, 1999.

[58] A. M. Niess, A. Hartmann, M. Grünert-Fuchs, B. Poch, and G. Speit, "DNA damage after exhaustive treadmill running in trained and untrained men," International Journal of Sports Medicine, vol. 17, no. 6, pp. 397-403, 1996.

[59] H. Miyazaki, S. Oh-ishi, T. Ookawara et al., "Strenuous endurance training in humans reduces oxidative stress following exhausting exercise," European Journal of Applied Physiology, vol. 84, no. 1-2, pp. 1-6, 2001.

[60] A. Musarò, S. Fulle, and G. Fanò, "Oxidative stress and muscle homeostasis," Current Opinion in Clinical Nutrition and Metabolic Care, vol. 13, no. 3, pp. 236-242, 2010.

[61] M. J. Jackson, "Reactive oxygen species and redox-regulation of skeletal muscle adaptations to exercise," Philosophical Transactions of the Royal Society B, vol. 360, no. 1464, pp. 2285-2291, 2005.

[62] S. K. Powers, E. E. Talbert, and P. J. Adhihetty, "Reactive oxygen and nitrogen species as intracellular signals in skeletal muscle," Journal of Physiology, vol. 589, no. 9, pp. 2129-2138, 2011.

[63] C. M. Bloor, "Angiogenesis during exercise and training," Angiogenesis, vol. 8, no. 3, pp. 263-271, 2005.

[64] W. W. Li, D. Tsakayannis, and V. W. Li, "Angiogenesis: a control point for normal and delayed wound healing," Cotemporary Surgery, vol. 27, no. 10, pp. 1293-1297, 2003.

[65] Y. Castier, R. P. Brandes, G. Leseche, A. Tedgui, and S. Lehoux, "p47phox-dependent NADPH oxidase regulates flow-induced vascular remodeling," Circulation Research, vol. 97, no. 6, pp. 533-540, 2005.

[66] S. Lehoux, "Redox signalling in vascular responses to shear and stretch," Cardiovascular Research, vol. 71, no. 2, pp. 269 279, 2006.

[67] S. Lehoux, C. A. Lemarié, B. Esposito, H. R. Lijnen, and A. Tedgui, "Pressure-induced matrix metalloproteinase-9 contributes to Early hypertensive remodeling," Circulation, vol. 109, no. 8, pp. 1041-1047, 2004.

[68] M. Milkiewicz, O. Hudlicka, J. Verhaeg, S. Egginton, and M. D. Brown, "Differential expression of Flk-1 and Flt-1 in rat skeletal muscle in response to chronic ischaemia: favourable effect of muscle activity," Clinical Science, vol. 105, no. 4, pp. 473-482, 2003.
[69] T. Gustafsson, A. Puntschart, L. Kaijser, E. Jansson, and C. J. Sundberg, "Exercise-induced expression of angiogenesisrelated transcription and growth factors in human skeletal muscle," American Journal of Physiology, vol. 276, no. 2, pp. H679-H685, 1999.

[70] P. G. Lloyd, B. M. Prior, H. T. Yang, and R. L. Terjung, "Angiogenic growth factor expression in rat skeletal muscle in response to exercise training," American Journal of Physiology, vol. 284, no. 5, pp. H1668-H1678, 2003.

[71] Z. Arany, S. Y. Foo, Y. Ma et al., "HIF-independent regulation of VEGF and angiogenesis by the transcriptional coactivator PGC-1 $\alpha$," Nature, vol. 451, no. 7181, pp. 1008-1012, 2008.

[72] T. L. Nemirovskaya, B. S. Shenkman, and V. B. Koshelev, "Exercise-induced hypoxia and structural and metabolic adaptation of skeletal muscle," Basic and Applied Myology, vol. 6, no. 8, pp. 441-445, 1998.

[73] X. Z. West, N. L. Malinin, A. A. Merkulova et al., "Oxidative stress induces angiogenesis by activating TLR2 with novel endogenous ligands," Nature, vol. 467, no. 7318, pp. 972-976, 2010.

[74] P. J. Adhihetty, I. Irrcher, A. M. Joseph, V. Ljubicic, and D. A. Hood, "Plasticity of skeletal muscle mitochondria in response to contractile activity," Experimental Physiology, vol. 88, no. 1, pp. 99-107, 2003.

[75] H. C. Lee and Y. H. Wei, "Mitochondrial biogenesis and mitochondrial DNA maintenance of mammalian cells under oxidative stress," International Journal of Biochemistry and Cell Biology, vol. 37, no. 4, pp. 822-834, 2005.

[76] V. Pesce, A. Cormio, F. Fracasso, A. M. S. Lezza, P. Cantatore, and M. N. Gadaleta, "Age-related changes of mitochondrial DNA content and mitochondrial genotypic and phenotypic alterations in rat hind-limb skeletal muscles," Journals of Gerontology A, vol. 60, no. 6, pp. 715-723, 2005.

[77] D. C. Wright, D. H. Han, P. M. Garcia-Roves, P. C. Geiger, T. E. Jones, and J. O. Holloszy, "Exercise-induced mitochondrial biogenesis begins before the increase in muscle PGC- $1 \alpha$ expression," Journal of Biological Chemistry, vol. 282, no. 1, pp. 194-199, 2007.

[78] I. Irrcher, V. Ljubicic, and D. A. Hood, "Interactions between ROS and AMP kinase activity in the regulation of PGC- $1 \alpha$ transcription in skeletal muscle cells," American Journal of Physiology, vol. 296, no. 1, pp. C116-C123, 2009.

[79] C. Scheele, S. Nielsen, and B. K. Pedersen, "ROS and myokines promote muscle adaptation to exercise," Trends in Endocrinology and Metabolism, vol. 20, no. 3, pp. 95-99, 2009.

[80] M. J. Jackson, S. Papa, J. Bolaños et al., "Antioxidants, reactive oxygen and nitrogen species, gene induction and mitochondrial function," Molecular Aspects of Medicine, vol. 23, no. 1-3, pp. 209-285, 2002.

[81] C. E. H. Stewart and P. Rotwein, "Growth, differentiation, and survival: multiple physiological functions for insulin-like growth factors," Physiological Reviews, vol. 76, no. 4, pp. 10051026, 1996.

[82] A. Philippou, A. Halapas, M. Maridaki, and M. Koutsilieris, "Type I insulin-like growth factor receptor signaling in skeletal muscle regeneration and hypertrophy," Journal of Musculoskeletal Neuronal Interactions, vol. 7, no. 3, pp. 208218, 2007.

[83] E. Barbieri and P. Sestili, "Reactive oxygen species in skeletal muscle signaling," Journal of Signal Transduction, vol. 2012, Article ID 982794, 17 pages, 2012.

[84] A. E. Handayaningsih, G. Iguchi, H. Fukuoka et al., "Reactive oxygen species play an essential role in IGF-I signaling and 
IGF-I-induced myocyte hypertrophy in C2C12 myocytes," Endocrinology, vol. 152, no. 3, pp. 912-921, 2011.

[85] A. L. Serrano, B. Baeza-Raja, E. Perdiguero, M. Jardí, and P. Muñoz-Cánoves, "Interleukin-6 is an essential regulator of satellite cell-mediated skeletal muscle hypertrophy," Cell Metabolism, vol. 7, no. 1, pp. 33-44, 2008.

[86] I. Kosmidou, T. Vassilakopoulos, A. Xagorari, S. Zakynthinos, A. Papapetropoulos, and C. Roussos, "Production of interleukin-6 by skeletal myotubes: role of reactive oxygen species," American Journal of Respiratory Cell and Molecular Biology, vol. 26, no. 5, pp. 587-593, 2002.

[87] F. Lluís, E. Perdiguero, A. R. Nebreda, and P. Muñoz-Cánoves, "Regulation of skeletal muscle gene expression by p38 MAP kinases," Trends in Cell Biology, vol. 16, no. 1, pp. 36-44, 2006.

[88] S. K. Powers, A. N. Kavazis, and J. M. McClung, "Oxidative stress and disuse muscle atrophy," Journal of Applied Physiology, vol. 102, no. 6, pp. 2389-2397, 2007. 


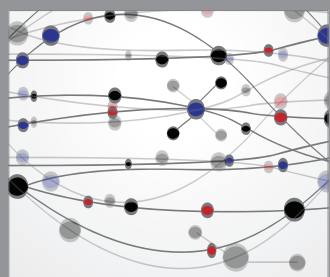

The Scientific World Journal
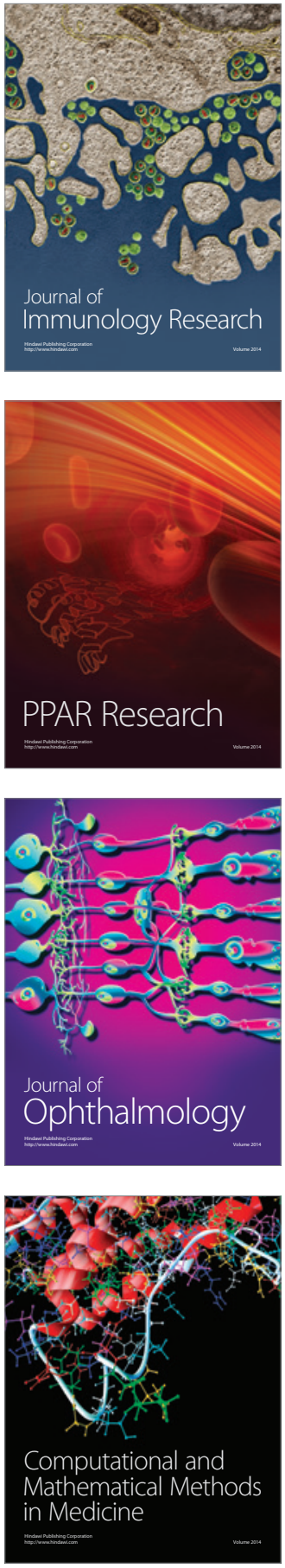

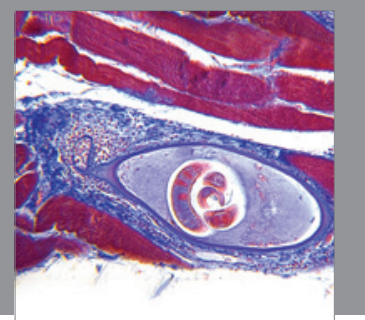

Gastroenterology

Research and Practice
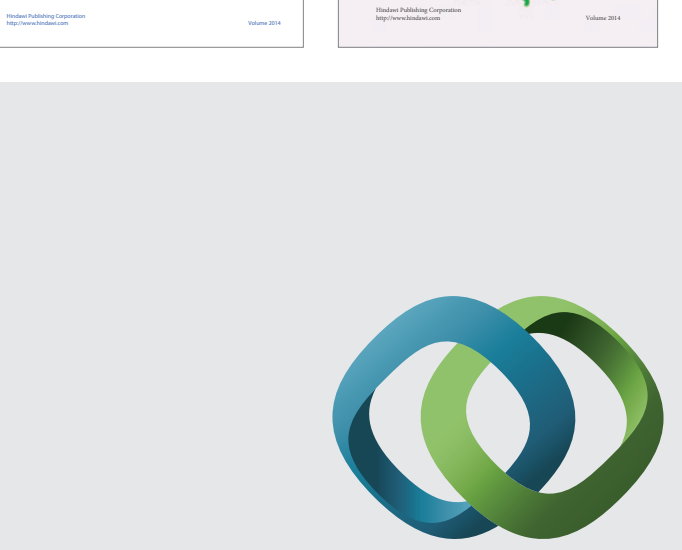

\section{Hindawi}

Submit your manuscripts at

http://www.hindawi.com
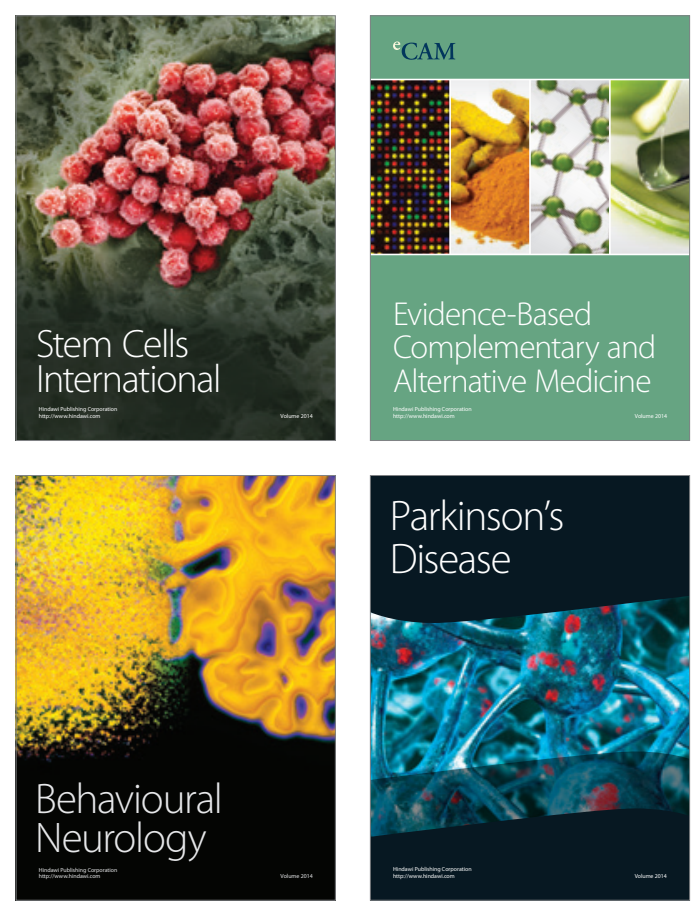

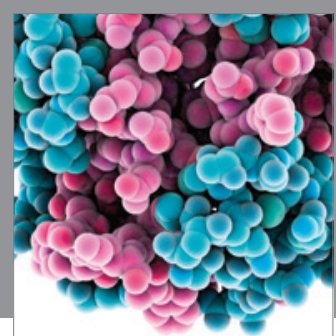

Journal of
Diabetes Research

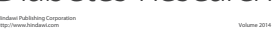

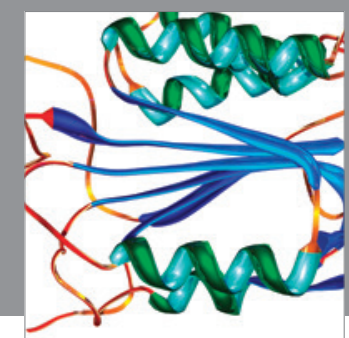

Disease Markers
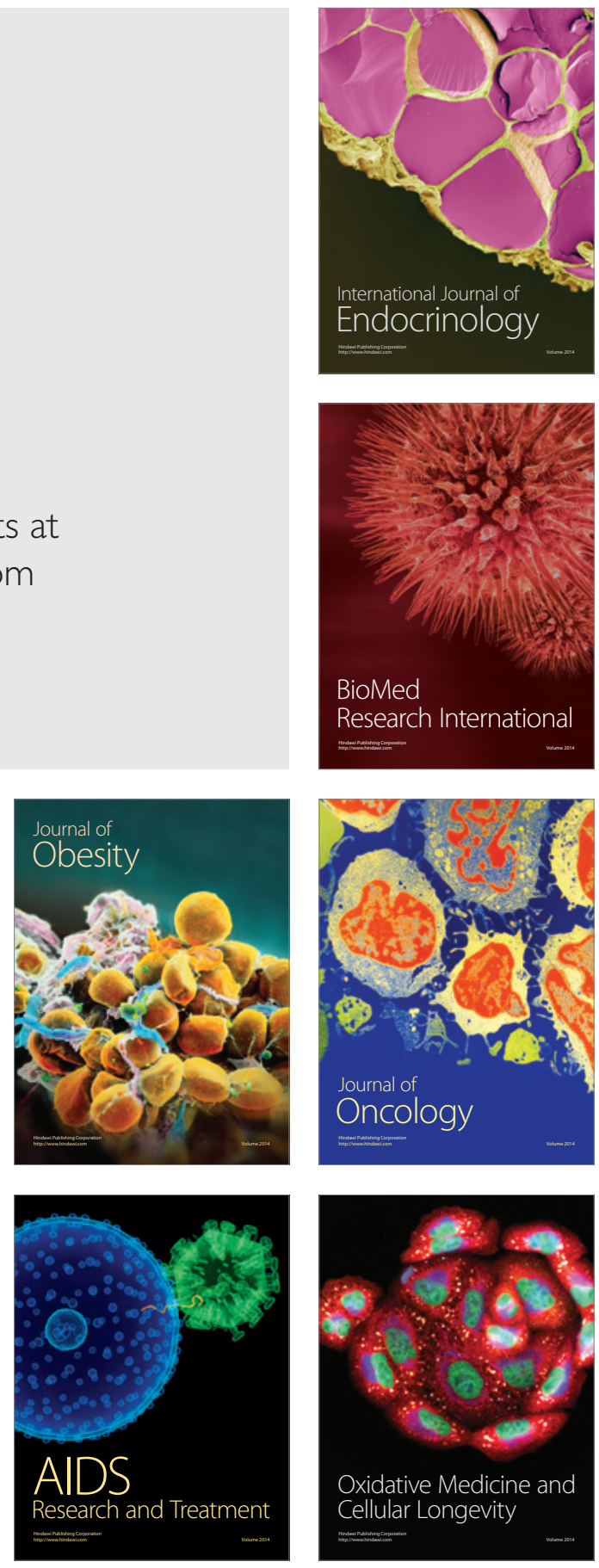